Trauma Berufskrankh 2012 • 14[Suppl 2]:149-153 DOI 10.1007/s10039-011-1798-9

Online publiziert: 8. Oktober 2011

(c) Springer-Verlag 2011

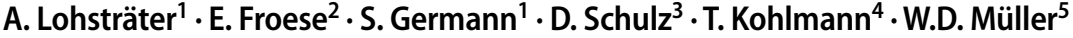 \\ ${ }^{1}$ VBG, Bezirksverwaltung Erfurt, Erfurt \\ ${ }^{2}$ VBG, Hauptverwaltung - Rehabilitationsstab, Hamburg \\ ${ }^{3}$ Sport- und Rehacentrum, Magdeburg \\ ${ }^{4}$ Abteilung Methoden der Community Medicine, \\ Institut für Community Medicine, Universität Greifswald, Greifswald \\ ${ }^{5}$ Abteilung für Orthopädie/Unfallchirurgie, $m$ \& i Fachklinik, Bad Liebenstein
}

\title{
Arbeitsplatzorientierte muskuloskelettale Rehabilitation (ABMR)
}

\section{In der gesetzlichen Unfallversicherung}

\section{Arbeitsplatzbezogene \\ muskuloskelettale Rehabilitation}

\section{Komplextherapeutische Rehabilitationsverfahren}

\section{Bisherige Verfahren}

In der gesetzlichen Unfallversicherung ist es seit ihrer Gründung Ziel der Rehabilitation, die Gesundheit und die Leistungsfähigkeit der Versicherten wiederherzustellen sowie den Betroffenen die Teilhabe am Arbeitsleben und am Leben in der Gemeinschaft möglichst schnell und dauerhaft wieder zu ermöglichen. Um dies zu erreichen, bedienen sich die Unfallversicherungsträger verschiedener komplextherapeutischer Rehabilitationsverfahren, wie der berufsgenossenschaftlichen stationären Weiterbehandlung (BGSW) und der erweiterten ambulanten Physiotherapie (EAP).

In diesen etablierten Verfahren der muskuloskelettalen Rehabilitation standen bisher struktur- und funktionsbezogene Therapieansätze im Vordergrund. Arbeitsplatz- oder erwerbstätigkeitsorientierte Behandlungsinhalte waren unterrepräsentiert. Um dieses Defizit zu beseitigen, wurde als manualisiertes und standardisiertes Gesamtkonzept die arbeitsplatzbezogene muskuloskelettale Rehabilitation (ABMR) entwickelt.
Ziel der ABMR ist es, während der medizinischen Rehabilitation nicht nur Funktions- und Strukturstörungen zu beseitigen oder zu kompensieren, sondern vielmehr auch konkret benötigte arbeitsrelevante Funktionsabläufe in die Therapie zu integrieren. Dies geschieht mit Hilfe einer spezifischen Arbeitsplatzorientierung, um eine ausreichende funktionale Belastbarkeit für die sich unmittelbar anschließende vollschichtige Rückkehr an den (bisherigen) Arbeitsplatz zu erreichen.

Wesentliche Bestandteile des Verfahrens sind die Erhebung und Dokumentation eines detaillierten, standardisierten Arbeitsplatzanforderungsprofils, um die im Hinblick auf die Unfallverletzung kritischen Belastungselemente des jeweiligen Arbeitsplatzes zu identifizieren, sowie die Erstellung eines Patientenfähigkeitsprofils mittels FCE-Analyse (FCE: "functional capacity evaluation") im Hinblick auf die kritischen Arbeitsplatzanforderungen z. B. mit dem EFL-Screeningtest (EFL: Evaluation funktioneller Leistungsfähigkeiten). Der Vergleich von Anforderungs- und Fähigkeitsprofil erlaubt die Identifizierung der vorhandenen körperlichen Defizite und das Festlegen der auf deren Beseitigung gerichteten Therapie bzw. Therapieelemente.

Die bislang angewandten Therapieinhalte werden durch die Folgenden zusätzlichen arbeitsplatzorientierten Therapieelemente ergänzt:

- Ergotherapie mit dem Schwerpunkt in der Arbeitstherapie,

- „work conditioning“, d. h. an den Arbeitsplatzanforderungen ausgerichtete medizinische Trainingstherapie (MTT) sowie

- „work hardening“ bzw. Arbeitssimulationstraining (durch ein speziell geschultes Team von Sportlehrern, Ergotherapeuten bzw. Krankengymnasten),

bei denen der Rehabilitand die physischen Behandlungsergebnisse aus der KG (Krankengymnastik), PT (Physiotherapie) und MTT in Übungen, die den Anforderungen der beruflichen Tätigkeit entsprechen, umsetzt.

Optional kann in Abstimmung mit der VBG (Verwaltungsberufsgenossenschaft) bei Patienten mit psychischen Auffälligkeiten ein Psychologe herangezogen werden.

Wesentliche Voraussetzung für die ABMR sind eine ausreichende medizinische Grundbelastbarkeit sowie die 

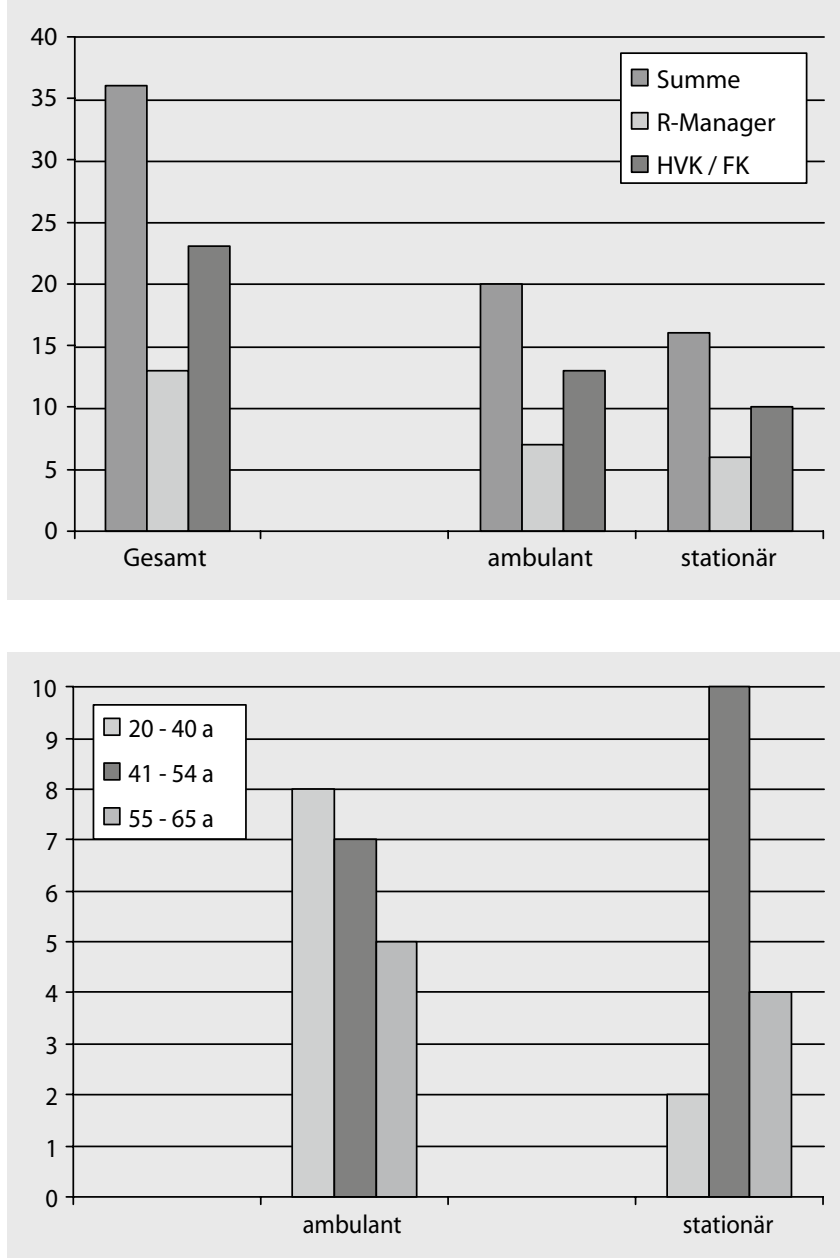

- die Gesamtdauer der Arbeitsunfähigkeit,

- das Outcome nach Arbeitsfähigkeit hinsichtlich einer evtl. Minderung der Erwerbsfähigkeit (MdE).

\section{Ergebnisse}

\section{Studiengröße}

Abb. 14 Verteilung der Zuweisung, HVKJ FK Heilverfahrenskontrolle/Fallkonferenz, $R$ Manager Rehabilitationsmanager

Abb. $2<$ Altersverteilung der Patienten, a Jahre
Prognose, möglichst ab AOMR-Beginn (AOMR: arbeitsplatzorientierte muskuloskelettale Rehabilitation) innerhalb der nachfolgenden 4 Wochen die Arbeitsfähigkeit zu erreichen.

Die ABMR erscheint insbesondere indiziert bei körperlich arbeitenden Menschen sowie Menschen mit spezifischen körperlichen Arbeitsbelastungen (einseitig monoton und/oder koordinativ beanspruchende Tätigkeiten) im Bereich der verletzten Körperregion.

Parallel zur Einführung dieses Verfahrens bei der VBG erfolgte eine Machbarkeitsuntersuchung durch eine prospektive Beobachtungsstudie.

\section{Material und Methode}

Die Studie wurde als prospektive Beobachtungsstudie durchgeführt. Eingeschlossen waren kaufmännische und gewerbliche Arbeitnehmer beiderlei Ge- schlechts im Alter von 20-65 Lebensjahren zum Unfallzeitpunkt.

Beobachtet wurde das 1. bis 3. Quartal 2010 in einer ambulanten (Magdeburg) und einer stationären Rehabilitationseinrichtung (Bad Liebenstein).

Gemessen bzw. ausgewertet wurden:

- die Anzahl der durchgeführten Maßnahmen im Beobachtungszeitraum je Einrichtung,

- durch wen die Zuweisung zur ABMR erfolgte,

- das Patientenalter,

- die ausgeübte Tätigkeit (kaufmännisch/gewerblich) zum Unfallzeitpunkt,

- die Verletzungsregion und die Diagnose,

- vorangegangene Komplextherapien und Behandlungseinheiten je Fall und Einrichtung,

- der Erfolg der ABMR im Sinne der Arbeitsfähigkeit nach Abschluss der Maßnahme,
In der Studie wurden insgesamt 42 ABMR-Maßnahmen analysiert. Davon wurden 22 ambulant und 20 stationär durchgeführt.

Von den 22 ambulanten ABMR-Verfahren konnten 2 Maßnahmen vorzeitig beendet werden. In einem Fall konnte durch den Profilvergleich nahezu sofort Arbeitsfähigkeit festgestellt werden; im zweiten Fall zeigte die AOMR, dass eine Revisionsoperation erforderlich war.

Von den 20 stationär begonnenen Maßnahmen wurden 4 vorzeitig beendet. In 2 Fällen war es nicht möglich, die Trainingsleistung auf das Niveau der vollschichtigen Arbeitsleistung zu steigern, sodass Maßnahmen zur Teilhabe am Arbeitsleben eingeleitet wurden. Bei einem Patienten wurde eine akutstationäre Behandlung notwendig. Bei einem weiteren Patienten stellte sich heraus, dass die notwendige medizinische Grundbelastbarkeit noch nicht gegeben war. Hier wurde zunächst eine BGSW durchgeführt.

Ausgewertet werden konnten 20 ambulant und 16 stationär durchgeführte arbeitsplatzorientierte muskuloskelettale Rehabilitationsmaßnahmen.

\section{Zuweisung zur ABMR}

Sie erfolgte entweder ausschließlich durch den Rehabilitationsmanager der VBG oder im Rahmen einer Heilverfahrenskontrolle/Fallkonferenz (HVK/FK) gemeinsam durch den hinzugezogenen Facharzt und den Rehabilitationsmanager, Einzelheiten hierzu zeigt $\bullet$ Abb. 1.

\section{Patientenalter, Geschlecht und ausgeübte Berufstätigkeit}

Die 22 Patienten in der ambulanten Einrichtung waren zum Unfallzeitpunkt im Durchschnitt 41,1 Jahre alt (20-60 Lebensjahre) und alle männlich. Die $20 \mathrm{~Pa}$ - 
tienten in der stationären Rehabilitationseinrichtung waren im Durchschnitt 49,0 Jahre alt (30-63 Lebensjahre). Es handelte sich um 1 Frau und 15 Männer. Die Altersverteilung für beide Einrichtungen ist in $\square$ Abb. 2 dargestellt.

An der ambulant durchgeführten ABMR nahmen 20 gewerbliche Arbeitnehmer teil, im Rahmen der stationären ABMR wurden 15 gewerbliche Patienten und 1 Patientin mit einer kaufmännischen Beschäftigung behandelt.

\section{Verletzungsregionen und Diagnosen}

Von den 20 ambulant behandelten Patienten hatten

- 11 Verletzungen der unteren Extremität,

- 7 Handverletzungen und

- 2 Wirbelsäulenverletzungen.

Bei den Verletzungen der unteren Extremität dominierten die Fersenbein- $(n=3)$ und Sprunggelenkverrenkungsbrüche $(n=3)$. Bei den Handverletzungen waren überwiegend Handwurzelfrakturen $(n=3)$ oder schwere Weichteilschäden $(n=2) \mathrm{zu}$ verzeichnen.

Die 16 stationären ABMR-Verfahren teilten sich wie folgt auf:

- 9 Verletzungen der unteren Extremität,

- 4 Beckenfrakturen,

- 2 Handverletzungen,

- 1 Verletzung der oberen Extremität.

Bei den Verletzungen der unteren Extremität überwogen die Fersenbein- $(n=3)$ und die gelenknahen Tibiabrüche $(n=5)$.

\section{Behandlungseinheiten und vorhergehende Komplextherapien}

Die ambulant durchgeführten ABMRVerfahren dauerten im Mittel 12,9 Behandlungstage (10-20 Tage). Vor der ABMR hatten 15 der 20 Patienten bereits eine andere komplextherapeutische Rehabilitation zur Herstellung der Grundbelastbarkeit absolviert. In 7 Fällen war dies eine BGSW, in 5 Fällen eine EAP, und in 3 Fällen waren sowohl eine BGSW als auch eine EAP erfolgt (mit 14-tägigem Verlauf). Zwischen den Maßnahmen la-

Trauma Berufskrankh 2012 · 14[Suppl 2]:149-153 DOI 10.1007/s10039-011-1798-9

(c) Springer-Verlag 2011

\section{A. Lohsträter · E. Froese · S. Germann · D. Schulz · T. Kohlmann · W.D. Müller}

\section{Arbeitsplatzorientierte muskuloskelettale Rehabilitation (ABMR). In der gesetzlichen Unfallversicherung}

\section{Zusammenfassung}

Die ABMR ist seit Mai 2011 ein Modul der Rehabilitation der Deutschen Gesetzlichen Unfallversicherung (DGUV). Wesentlicher Bestandteil sind die Erhebung eines detaillierten, standardisierten funktionellen Arbeitsplatzanforderungsprofils sowie die Erstellung eines Patientenfähigkeitsprofils. Ein Vergleich dieser Profile erlaubt die Identifizierung der vorhandenen körperlichen Defizite und das Festlegen der auf ihre Beseitigung gerichteten Therapie. Die Wirksamkeit der ABMR wurde mittels einer prospektiven Beobachtungsstudie überprüft. Eingeschlossen wurden kaufmännische und gewerbliche Arbeitnehmer beiderlei Geschlechts im Alter von 2065 Lebensjahren zum Unfallzeitpunkt. Gemessen wurden verschiedene Rehabilita- tionsprozessparameter, die Ergebnisse hinsichtlich des Wiedereintritts der Arbeitsfähigkeit sowie die verbleibende MdE (Minderung der Erwerbsfähigkeit). Nach Abschluss der ABMR wurde unmittelbar Arbeitsfähigkeit für die jeweils zuletzt ausgeübte Erwerbstätigkeit erreicht. Die Zahl der Fälle mit einer verbleibenden MdE war niedrig. Die ABMR erwies sich als spezifische Therapieform mit großem Erfolg, die eine schnellstmögliche Rückkehr an den Arbeitsplatz fördert. Wünschenswert sind weitere Studien zum nachhaltigen Effizienznachweis.

\section{Schlüsselwörter}

Unfallversicherung $\cdot$ Rehabilitation . Arbeitsplatz $\cdot A B M R \cdot$ Wiedereingliederung

\section{Workplace-oriented musculoskeletal rehabilitation. In the German statutory accident insurance}

\section{Abstract}

As of May $3^{\text {rd }}$, 2011, workplace-related muscular skeletal rehabilitation has been a rehabilitation module within the German Statutory Accident Insurance (DGUV). Essential components of the procedure include compiling and documenting detailed standardized functional workplace profiles. A comparison of these profiles enables existing physical deficits to be identified and adequate deficit-orientated therapy to be planned. The effectiveness of workplace-related musculoskeletal rehabilitation was measured by means of a prospective monitoring study. Commercial and industrial employees (both male and female) aged between 20 and 65 years at the time of their accident were included in the study. Numerous rehabilitation process parameters, the results of re-entry to work as well as residual reductions in work capacity were evaluated. Immediately following workplace-related musculoskeletal rehabilitation, work capacity for the last job performed could be achieved. The number of cases of a reduction in work capacity was low. Workplace-related musculoskeletal rehabilitation proved to be a highly successful specific therapy, supporting re-entry to work within a minimum period of time. Further, possibly randomized studies for sustainable evidence of efficiency are desirable.

\section{Keywords}

Accident insurance $\cdot$ Rehabilitation .

Workplace . Workplace-related

musculoskeletal rehabilitation · Re-entry gen im Mittel 16,2 Tage (2-59 Tage). Bei 5 Patienten wurde die ABMR nach längerem Verlauf mit monotherapeutischen Maßnahmen durchgeführt.

Die stationäre ABMR dauerte im Mittel 17,6 Tage (12-30 Tage). Vor der ABMR hatten 11 Patienten eine BGSW (9 Patienten), eine EAP (1 Patient) oder eine BGSW und EAP (1 Patient) erhalten. Die Zeit zwischen den Maßnahmen betrug im Mittel 36,5 Tage (2-80 Tage). Bei 5 Patien- ten wurde die ABMR nach längerem Verlauf mit monotherapeutischen Maßnahmen durchgeführt.

\section{Arbeitsfähigkeit, Dauer und Outcome}

In allen 36 durchgeführten ABMR-Verfahren konnte nach Abschluss der Maßnahmen unmittelbar Arbeitsfähigkeit für 


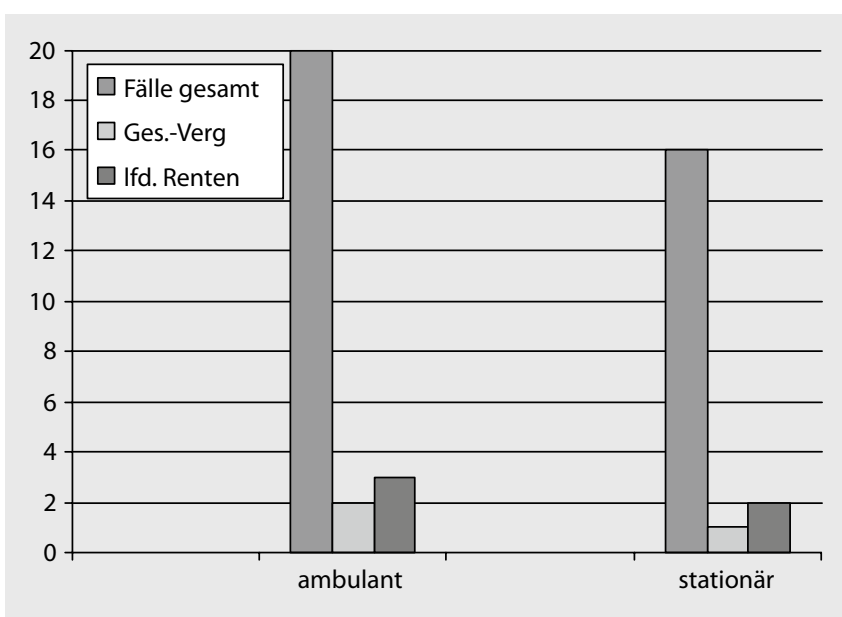

Abb. $3<$ Outcomeverteilung, Ges.-Verg Gesamtvergütungen, Ifd. Renten laufende Renten die jeweils zuletzt ausgeübte Erwerbstätigkeit festgestellt werden.

Die durchschnittliche Gesamtdauer der Arbeitsunfähigkeit betrug bei den ambulant rehabilitierten Patienten 188,3 Tage (70-495 Tage); bei den stationär Rehabilitierten im Mittel 214,9 Tage (86-407 Tage). Dies korreliert mit dem Verletzungsausmaß.

Das Outcome im Sinne einer vorhandenen Minderung der Erwerbsfähigkeit (MdE) zeigt - Abb. 3. Dabei waren sowohl die Anzahl der Gesamtvergütungen als auch der laufenden Renten im Verhältnis zur beobachteten Patientenzahl gering. Bisherige Publikationen ergaben je nach Fraktur und Verletzungstyp für bis zu 75\% der untersuchten Kohorten Rentenzahlungen $[11,12,17]$.

\section{Diskussion}

Die Verfahren der Rehabilitation zum Erreichen der Ziele in der gesetzlichen Unfallversicherung, die Gesundheit und die Leistungsfähigkeit der Versicherten wiederherzustellen sowie den Betroffenen die Teilhabe am Arbeitsleben und am Leben in der Gemeinschaft schnell und dauerhaft wieder zu ermöglichen, unterliegen aktuell einem Wandel. Dabei nimmt in jüngerer Zeit die arbeitsplatzorientierte bzw. -bezogene Rehabilitation bei allen Rehabilitationsträgern einen zunehmenden Stellenwert ein.

Strukturelle Veränderungen des Bewegungssystems nach Unfällen können in der Regel nicht rückgängig gemacht werden. Der Körper ist aber in der Lage, durch Training andere Strukturen so zu verbessern, dass Funktionsdefizite minimiert, Kompensationsbewegungen erlernt und der Umgang mit den Schmerzen („coping“) verbessert wird [2]. Eine entscheidende Aufgabe hinsichtlich der Motivation hierfür liegt beim Verunfallten selbst. Er muss das Angstvermeidungsverhalten („fear avoidance belief“) überwinden $[10,15]$.

Ausgehend von den dargestellten Prämissen ergeben sich für die bekannten komplextherapeutischen Rehabilitationsverfahren Schnittstellen [3]:

- Die medizinische Rehabilitation ist zu stark auf die allgemeine Funktionsfähigkeit und die allgemeine Leistungsfähigkeit der Rehabilitanden ausgerichtet.

- In der Regel liegen zu Beginn der Rehabilitation nur unzureichende Informationen über den konkreten Arbeitsplatz vor.

- Die Abschlussbeurteilung im Rehabilitationsabschlussbericht ist für die konkrete Einschätzung der aktuellen Leistungsfähigkeit/Arbeitsfähigkeit oft wenig hilfreich.

Untersuchungen zur Wirksamkeit spezifischer Verfahren im Sinne einer arbeitsplatzorientierten bzw. -bezogenen Rehabilitation wurden vereinzelt veröffentlicht $[14,16]$. Ein zweistufiges Verfahren einer einzelnen Rehabilitationsklinik zur arbeitsplatzorientierten Rehabilitation von Rückenschmerzpatienten wurde im Rahmen des 11. rehabilitationswissenschaftlichen Kolloquiums der DRV 2002 vorgestellt [14]. Der Einsatz von Arbeitsplatzanforderungsprofilen oder durchge- führte Profilvergleiche zwischen Fähigkeits- und Anforderungsprofil wurden nicht beschrieben. Eine arbeitsplatzbezogene Rehabilitation im Rahmen einer BGSW bzw. einer komplexen stationären Rehabilitation (KSR) beschrieb Wernicke [16]. Er postulierte ein vierstufiges Verfahren mit extern begleiteter Arbeitsund Belastungserprobung. Auch in diesem fehlte der Einsatz von Arbeitsplatzanforderungsprofilen oder durchgeführter Profilvergleiche im oben dargestellten Sinne.

Eine wesentliche Grundlage zur Entwicklung des ABMR-Konzepts lieferte eine Reihe von vorangegangenen Untersuchungen, deren Schwerpunkte die ergebnisorientierte Optimierung von Rehabilitationsverfahren waren. Dabei zeigte sich, dass der entscheidende Schritt zur Verbesserung der Ergebnisqualität der Rehabilitationsverfahren die systematische Einführung eines arbeitsplatzbezogenen Profilvergleichs (Arbeitsplatzanforderungs- vs. Patientenfähigkeitsprofil) darstellen würde $[7,8,9]$. Den so festgestellten Unterschied der kritischen Belastungselemente des jeweiligen Arbeitsplatzes zu dem Fähigkeitsprofil des Patienten gilt es, zu rehabilitieren, wobei die konkret benötigten arbeitsrelevanten Funktionsabläufe in die Therapie zu integrieren sind.

Zur systematischen Beurteilung der funktionalen Leistungsfähigkeit am Arbeitsplatz etablierten sich in der Praxis verschiedene Testverfahren (z. B. ER$\mathrm{GOS}^{\circledast}$ und EFL), die eine valide Aussage über die weitere berufliche Prognose und den voraussichtlichen Wiedereintritt der Arbeitsfähigkeit erlauben. Beim ERGOS $^{\circledR}$ wird ein spezieller computergesteuerter Arbeitssimulator eingesetzt, der durch medizinische Beurteilung ergänzt wird [5]. Häufiger wird in den Rehabilitationseinrichtungen das von Isernhagen [4] entwickelte Verfahren der Evaluation der funktionellen Leistungsfähigkeit (EFL) verwendet $[4,6]$, da es deutlich weniger technischen Aufwand erfordert und leichter zur Erfolgskontrolle von Fortschritten beim Arbeitstraining („work hardening“) eingesetzt werden kann.

Das hier vorgestellte Konzept wurde 2009 entwickelt; die Projektphase fand vom 4. Quartal 2009 bis zum Ende des 3. Quartals 2010 statt. Die Deutsche Renten- 
versicherung Bund führt ihre Modellphase der medizinisch-beruflich orientierten Rehabilitation (MBOR) in einer 15-monatigen Projektzeit, begonnen im Oktober, 2010 durch [1].

Die Analyse der hier vorgestellten Pilotphase der ABMR zeigte einen strukturierten Behandlungspfad über den gesamten Verlauf, wobei das Arbeitsplatzanforderungsprofil bereits zu Beginn der multimodalen Komplextherapie vorliegt. Dieses wird regelhaft beim Arbeitgeber abgefragt. Denn vereinzelte Voruntersuchungen ergaben, dass die Einschätzung eines Rehabilitanden bezüglich der Anforderungen an seinen Arbeitsplatz durchaus nicht mit den Erhebungen des Arbeitgebers bzw. Betriebsarztes korreliert. In bis zu 25\% der untersuchten Verläufe stufte der Patient die Belastung im Vergleich als geringer ein [13].

Regelhafter Bestandteil des Verfahrens sind zu Beginn der ABMR die Erhebung und Dokumentation einer detaillierten, standardisierten Tätigkeitsanalyse, um die kritischen Belastungselemente des jeweiligen Arbeitsplatzes zu identifizieren, sowie die Anfertigung eines $\mathrm{Pa}$ tientenfähigkeitsprofils durch ein Screening der Leistungsfähigkeit hinsichtlich der kritischen Arbeitsplatzanforderungen mittels eines FCE-Systems. Damit werden Grundanforderungen an ein manualisiertes Verfahren erfüllt.

Die Verletzungsschwere in den untersuchten Einrichtungen zeigte sich erwartungsgemäß mit unterschiedlichen Schwerpunkten hinsichtlich Diagnosen bzw. Körperregionen, die dem jeweiligen Anspruch an eine stationäre oder ambulante arbeitsplatzorientierte muskuloskelettale Rehabilitation entsprachen.

Hinsichtlich der Zusteuerung der Patienten zum Verfahren besteht Optimierungspotenzial. Dies dürfte dem Bekanntheitsgrad eines Verfahrens in einer Pilotphase geschuldet sein. Mit Einführung der AOMR sind die Prozessabläufe bei den an der Rehabilitation Beteiligten zu schulen und weiterzuentwickeln. Festzustellen bleibt, dass sowohl behandelnde Ärzte als auch die Rehabilitationsmanager des Trägers insbesondere an den Schnittstellen erheblichen Einfluss auf den Verlauf des Prozesses haben.

\section{Fazit für die Praxis}

Die ABMR lässt als spezifische Therapieform mit hohem Erfolgspotenzial charakterisieren. Mit einer arbeitsplatzorientierten muskuloskelettalen Rehabilitation ist der "return to work" offenbar eher möglich als allein mit den bisher praktizierten komplextherapeutischen, rein funktionell ausgerichteten Rehabilitationsmaßnahmen. Weiterführende wissenschaftliche Studien zum Nachweis der Effizienzvorteile des ABMR-Verfahrens sind notwendig.

\section{Korrespondenzadresse}

\section{Dr. A. Lohsträter}

VBG, Bezirksverwaltung Erfurt, Koenbergkstraße 1, 99084 Erfurt Axel.Lohstraeter@vbg.de

Interessenkonflikt. Der korrespondierende Autor gibt an, dass kein Interessenkonflikt besteht.

\section{Literatur}

1. DRV-Bund (2010) Anforderungsprofil zur Durchführung der medizinisch-beruflich orientierten Rehabilitation - somatische Erkrankungen 2010. Deutsche Rentenversicherung Bund, Berlin, http:// www.deutsche-rentenversicherung-bund.de/SharedDocs/de/Inhalt/Zielgruppen/01_sozialmedizin_ forschung/05_konzepte_systemfragen/konzepte/ mbor.html?nn=37110

2. Föh KP (2007) Effekte einer ambulanten arbeitsplatzbezogenen Rehabilitation für Beschäftigte in der Flugzeugindustrie. Med. Dissertation, Universität Lübeck

3. Gutenbrunner C (2007) JobReha - ein innovatives Konzept für die arbeitsplatzorientierte Rehabilitation. Medizinische Hochschule Hannover, http:// www.mh-hannover.de/fileadmin/organisation/ ressort_krankenversorgung/downloads/innovationsgipfel/ig6/2_Gutenbrunner_JobReha.pdf

4. Isernhagen SJ (1988) Functional capacity evaluation. In: Isernhagen SJ (Hrsg) Work injury: management and prevention. Aspen, Gaithsburg S 139174

5. Kaiser H, Kersting M (2000) Der Stellenwert des Arbeitssimulationsgerätes ERGOS als Bestandteil der leistungsdiagnostischen Begutachtung. Rehabilitation 39:175-184

6. Kaiser H, Kersting M, Schian H-M et al (2000) Der Stellenwert des EFL-Verfahrens nach Isernhagen in der medizinischen und beruflichen Rehabilitation. Rehabilitation 39:297-306

7. Müller WD, Bak P, Maier V et al (2005) Ergebnisorientierte Optimierung der Reha-Konzepte bei berufstätigen Patienten mit rezidivierenden Rückenschmerzen - eine kontrollierte klinische Studie. 14. Rehabilitationswissenschaftliches Kolloquium, Hannover
8. Müller WD, Maier V, Bak P, Smolenski UC (2006) Verzahnung zwischen medizinischer Rehabilitation, beruflicher Reintegration bei Beschäftigten der Automobilindustrie mit Rücken- und Gelenkschmerzen. Phys Med Rehab Kuror 16:149-154

9. Müller WD, Lohsträter A, Kropf U et al (2008) Optimierung der berufsgenossenschaftlichen stationären Weiterbehandlung (BGSW) durch systematischen Einsatz von Assessmentinstrumenten. Phys Med Rehab Kuror 18:270-278

10. Philips HC, Jahanshahi M (1986) The components of pain behaviour report. Behav Res Ther 24:117125

11. Prokop A, Müller S, Warnke T, Rehm KE (2007) Problem Fersenbeinfraktur - Nachuntersuchungsergebnisse von 42 isolierten Frakturen. Trauma Berufskrankh 9:315-318

12. Radek E, Köhler T, Münch K et al (2003) Verletztenrenten auf unbestimmte Zeit nach distalen Radiusfrakturen. Trauma Berufskrankh 5:253-259

13. Riedl G, Haase I, Birkholz B (2002) Wie zuverlässig ist die Einschätzung eines Rehabilitanden bezüglich der Anforderungen an seinen Arbeitsplatz? 11. Rehabilitationswissenschaftliches Kolloquium des VDR, Tagungsband. VDR, Frankfurt am Main, S 4344

14. Schumacher HL (2002) B A C K - Berufsorientierte Rehabilitation für Arbeitsunfähige oder von Arbeitsunfähigkeit bedrohte Patienten mit chronischen Kreuzschmerzen. 11. Rehabilitationswissenschaftliches Kolloquium des VDR, Tagungsband. VDR, Frankfurt am Main, S 44-45

15. Waddell G, Newton M, Henderson I et al (1993) A fear-avoidance beliefs questionaire $(\mathrm{FABQ})$ and the role of fear-avoidance beliefs in chronic lowback pain and disability. Pain 52:157-168

16. Wernicke F (2007) Arbeitsplatzbezogene Rehabilitation im Rahmen der BGSW/KSR. Trauma Berufskrankh [Suppl 1] 9:S98-S102

17. Zwipp H (2009) Verletzungen des Fußes. Trauma Berufskrankh [Suppl 3] 11:249-260 